\title{
Melatonin inhibits colon cancer RKO cell migration by downregulating Rho-associated protein kinase expression via the p38/MAPK signaling pathway
}

\author{
ZHEN LIU $^{1 *}$, DUOBING ZOU ${ }^{2 *}$, XIAOPING YANG ${ }^{1 *}$, XIAOLONG XUE $^{1}$ \\ LI ZUO $^{1,3}$, QING ZHOU ${ }^{1,3}$, RUOLEI HU ${ }^{1,3}$ and YUAN WANG ${ }^{1,3}$ \\ ${ }^{1}$ Laboratory of Molecular Biology, and Department of Biochemistry, Anhui Medical University, Hefei, Anhui 230032; \\ ${ }^{2}$ Laboratory of Stem Cell Transplantation, Ningbo First Hospital, Ningbo, Zhejiang 315000; ${ }^{3}$ Key Laboratory of \\ Gene Research of Anhui Province, Hefei, Anhui 230032, P.R. China
}

Received February 13, 2017; Accepted August 25, 2017

DOI: $10.3892 / \mathrm{mmr} .2017 .7836$

\begin{abstract}
Melatonin is predominately produced and secreted by the pineal gland, and inhibits cell growth in various cancer cell lines such as colorectal cancer. However, the precise mechanisms involved have not been fully elucidated. In the present study, the potential molecular mechanism underlying the efficacy of melatonin on migration in RKO colon cancer cells was investigated. The effects of melatonin and H-1152, a selective inhibitor of Rho-associated protein kinase (ROCK), on the migration of RKO cells were analyzed by an in vitro wound healing assay. The localization of zonula occludens-1 (ZO-1) and occludin were observed by immunofluorescence. Reverse transcription-quantitative polymerase chain reaction (qPCR) was performed to analyze the relative mRNA levels of ROCK, ZO-1 and occludin. In addition, western blot analysis was implemented to examine the expression of ROCK, phospho (p)-myosin phosphatase targeting subunit 1 (MYPT1), p-myosin light chains (MLC) and p-p38. The results revealed that the expression levels of ROCK2, p-MYPT1 and p-MLC in RKO cells were decreased, and the membrane protein expression of ZO-1 and occludin increased when the cells were treated with melatonin. qPCR demonstrated that melatonin downregulated $R O C K 2$ gene expression, and upregulated the expression of the ZO-1 and occludin genes. The levels of ZO-1 and occludin localized in the tight junctions
\end{abstract}

Correspondence to: Professor Ruolei Hu or Professor Yuan Wang, Laboratory of Molecular Biology, and Department of Biochemistry, Anhui Medical University, Mailbox 18, 81 Meishan Road, Hefei, Anhui 230032, P.R. China

E-mail: shenghuahrl@163.com

E-mail: wangyuan@ahmu.edu.cn

${ }^{*}$ Contributed equally

Key words: melatonin, human colon cancer, Rho-associated protein kinase, phospho-myosin phosphatase targeting subunit 1, migration were markedly increased in the immunofluorescence assay. In addition, the phosphorylation levels of p38 were reduced when the cells were treated with melatonin, and treatment with H-1152 downregulated p38 phosphorylation. The results indicated that melatonin may inhibit the migration of $\mathrm{RKO}$ colon cancer cells by downregulating ROCK expression via the $\mathrm{p} 38 /$ mitogen-activated protein kinase signaling pathway.

\section{Introduction}

Despite efforts to identify and clinically apply more effective anticancer agents, human colon cancer remains one of the most frequent cancer types worldwide, as well as in China (1). This is partly due to the limitations associated with chemotherapy as a result of drug resistance, and tumor recurrence and metastasis (2). Thus, considering the limitations associated with chemotherapy, the identification and development of novel therapeutic strategies are urgently required in order to prolong the survival of patients with colon cancer.

Melatonin (5-methoxy-N-acetyltryptamine) is an endogenous indole compound primarily secreted by the pineal gland (3). Previous studies have demonstrated that melatonin regulates biological activities, including circadian rhythm regulation, seasonal changes, sleep, reproduction and cardiovascular functions $(3,4)$. In addition, melatonin increases the anticancer effects of some chemotherapeutics by regulating a number of signal transduction pathways that are associated with cancer cell proliferation, apoptosis and migration (5-7). Previous studies have also revealed that melatonin inhibits ruffle formation in RKO colon cancer cells, and modulates microtubule and microfilament structure formation in epithelial cells, thereby suppressing cell migration $(8,9)$. Ordoñez et al $(10)$ confirmed that inhibition of the nuclear factor-kB signaling pathway contributed to the melatonin-induced suppression of HepG2 liver cancer cell migration and invasion.

Cell migration is critical for the invasion of surrounding tissues and in turn, into blood or lymph; it is therefore also important in the formation of metastases. Many of these processes require cell motility, which is driven by cycles of actin polymerization, cell adhesion and actomyosin 
contraction (11). Tumor cells, particularly those with high metastatic potential, often exhibit a loss of tight junctions (TJ). TJs are complexes comprised of multiple proteins, including occludin, claudins and zonula occludens-1 (ZO-1), which regulate the paracellular flux or permeability between adjacent cells (12). Downregulation of ZO-1 and occludin proteins have been associated with the migration and invasion of cancer cells $(13,14)$. In addition, previous findings have shown that cytoskeletal contraction, regulation of tight junction barrier function and the disruption of tight junction structure, are induced by the phosphorylation of myosin light chains (MLC) (15). MLCs are believed to be involved in the generation of the contractile force used for cell migration. Zou et al (8) also identified that melatonin inhibited the phosphorylation of MLC by downregulating the MLC kinase (MLCK) and p38 mitogen-activated protein kinase (MAPK) signaling pathway. However, Rho-associated protein kinase (ROCK) can phosphorylate the myosin phosphatase targeting subunit (MYPT), thereby inactivating MLC phosphatase, which results in the inhibition of the dephosphorylation of MLC (16). Therefore, inhibition of MLC phosphorylation may be a result of ROCK downregulation. ROCKs belong to the AGC family of serine-threonine kinases, and mainly regulate the structure and movement of cells by acting on the cytoskeleton. The MYPT, as the protein phosphatase-1-binding component, is a critical component of the myosin phosphatase complex (17). A previous study revealed that ROCK controls cell polarity in neutrophils and enhances actomyosin contractility (18). ROCK inhibition has also been demonstrated to activate Rac in Swiss 3T3 cells and increase membrane ruffling in HUVECs $(19,20)$. However, the inhibition of myosin phosphatase, and not ROCK inhibition, increased MLC phosphorylation and inhibited cell migration in fibroblasts (21). Thus, ROCK activation may decrease the migration of RKO colon cancer cells. In addition, inhibiting ROCK also suppressed the phosphorylation of p38 MAPK following interleukin-1 stimulation (22). The MAPK signaling pathway regulates TJ paracellular transport by modulating the expression of TJ proteins and thus, altering the molecular structure (16). These observations suggested that, within the different signaling pathways, ROCK, ZO-1 and occludin may control non-muscle cell motility. In addition, the MAPK signaling pathways, which include extracellular signal-regulated kinase (ERK), c-JUN N-terminal kinase (JNK) and p38 kinase, serve pivotal roles in cell proliferation, migration and apoptosis in mammals (23). The p38 signaling pathway has been associated with the regulation of important processes in colon cancer cells, including apoptosis, migration and proliferation $(24,25)$. A previous study has also indicated that melatonin may possess anti-invasive/anti-metastatic actions that involve the inhibition of the p38 MAPK signaling pathway in breast cancer (26).

However, it is unknown whether melatonin can suppress the migration of RKO cells via the phosphosphorylated (p)-p38 signaling pathway by inhibiting ROCK and/or inducing the expression of TJ proteins. Therefore, the aim of the present study was to investigate the inhibitory effect of melatonin on the migration of RKO cells. In addition, the expression of p-MYPT1, ROCK, p-MLC, ZO-1, occludin and p-p38 in the signal transduction pathway were assessed.

\section{Materials and methods}

Reagents. Melatonin was provided by the School of Pharmacy, Anhui Medical University (Anhui, China), and was dissolved in DMSO prior to addition to the complete cell culture medium. 3-(4,5-Dimethylthiazol-2-yl)-2,5-diphenyl tetrazolium bromide (MTT) and DMSO were obtained from Sigma-Aldrich; Merck KGaA (Darmstadt, Germany). The ROCK inhibitor, 5-[[(2s)-hexahydro-2-methyl-1H-1,4-diazepin-1-yl] sulfonyl]-4-methyl-isoquinoline, dihydrochloride (H-1152) was purchased from Cayman Chemical Company (Ann Arbor, MI, USA), and was dissolved in DMSO at the stock concentration of $10 \mathrm{mM}$. The specific p38 MAPK inhibitor SB203580 and the protein kinase C (PKC) activator phorbol 12-myristate 13-acetate (PMA) were purchased from Cayman Chemical Company. PMA and SB203580 were dissolved in DMSO with concentrations of 16 and $2 \mathrm{mM}$, respectively, and stored at $-20^{\circ} \mathrm{C}$. Dulbecco's modified Eagle's medium (DMEM) was obtained from Gibco; Thermo Fisher Scientific, Inc. (Waltham, MA, USA). Fetal bovine serum (FBS) was obtained from the Zhejiang Tianhang Biotechnology Co., Ltd. (Zhejiang, China). Primary antibodies (used for western blotting and immunofluorescence) against ROCK1 (cat. no. sc-17794), ROCK2 (cat. no. sc-1851), MLC (cat. no. sc-48414), p-p38 (cat. no. sc-7675-R), p38 (cat. no. sc-7149), ZO-1 (cat. no. sc-8147), occludin (cat. no. sc-8144), MYPT1 (cat. no. sc-514261), p-MYPT1 (cat. no. sc-17432), $\beta$-actin (cat. no. sc-47778) and $\mathrm{Na}^{+} / \mathrm{K}^{+}$-ATPase (cat. no. sc-21712) were purchased from Santa Cruz Biotechnology, Inc. (Dallas, TX, USA). The antibody against p-MLC (cat. no. 3674) was purchased from Cell Signaling Technology, Inc. (Danvers, MA, USA). The donkey anti-goat immunoglobulin (Ig)G fluorescein isothiocyanate (FITC)-conjugated secondary antibody and DAPI were obtained from Beijing Zhongshan Golden Bridge Biotechnology Co., Ltd. (Beijing, China). All the secondary antibodies (goat anti-mouse IgG antibody, cat. no. AP124P; rabbit anti-goat IgG antibody, cat. no. AP106P; goat anti-rabbit IgG antibody, cat. no. AP132P) were purchased from EMD Millipore (Billerica, MA, USA). The enhanced chemiluminescence (ECL) reagent was purchased from Pierce; Thermo Fisher Scientific, Inc. The bicinchoninic acid kit was obtained from Beyotime Institute of Biotechnology (Haimen, China).

Cell lines. The human RKO colon cancer cell line was purchased from the American Type Culture Collection (Manassas, VA, USA). Cells were cultured in high glucose DMEM culture media supplemented with $10 \% \mathrm{FBS}, 40 \mathrm{U} / \mathrm{ml}$ penicillin and $100 \mathrm{U} / \mathrm{ml}$ streptomycin at $37^{\circ} \mathrm{C}$ in a humidified atmosphere containing $5 \% \mathrm{CO}_{2}$ throughout the present study. Cells were grown to $70 \%$ confluency prior to the start of the experiment.

Wound healing assay. Cells were seeded in 12-well plates at a density of $6 \times 10^{4}$ cells/well and incubated at $37^{\circ} \mathrm{C}$ until cells grew to $100 \%$ confluency. Each well was then manually scratched with a sterile $200 \mu \mathrm{l}$ pipette tip to create the wound, and incubated at $37^{\circ} \mathrm{C}$ with melatonin $(2.5 \mathrm{mM})$ or $\mathrm{H}-1152$ (final concentration, $10 \mu \mathrm{M}$ ), alone or in combination. Images of the gap between the two cell edges at the same position on the wound were captured at 0,24 and $48 \mathrm{~h}$ using an inverted 
phase contrast microscope at a magnification of x100 (Leica DMI3000 B). The width of the wound was measured using Quantity One software (version 4.6.2; Bio-Rad Laboratories, Inc., Hercules, CA, USA) and the following calculation was applied: Half of migration distance $=($ end distance - start distance) $/ 2$.

Immunofluorescence assay. RKO cells were seeded in a 12-well Costar ${ }^{\circledR}$ cell culture cluster dish at a density of $6 \times 10^{4}$ cells/well with sterile coverslips placed in the wells prior to seeding and cultured at $37^{\circ} \mathrm{C}$. Once the cells formed a monolayer, they were treated with $2.5 \mathrm{mM}$ melatonin, or $0.1 \%$ DMSO as the control group, daily for 10 days; medium containing the different treatments was replaced every day. Following treatment, the cells were washed three times with 1X PBS and fixed with 4\% paraformaldehyde for $30 \mathrm{~min}$ at room temperature. Cells were then washed again with PBS and blocked with blocking buffer (PBS containing 1\% bovine serum albumin; Sigma-Aldrich; Merck $\mathrm{KGaA}$ ) for $2 \mathrm{~h}$ at room temperature. The coverslips with cells were incubated with goat anti-human ZO-1 and occludin (both 1:50) primary antibodies overnight at $4^{\circ} \mathrm{C}$. Subsequently, the coverslips were washed and incubated with a donkey anti-goat IgG FITC-conjugated secondary antibody (1:100) for $2 \mathrm{~h}$ at room temperature away from light. The cells were washed, incubated with DAPI for $5 \mathrm{~min}$ at room temperature, washed again, mounted with aqueous-based anti-fade mounting medium and then fixed with colorless nail polish on microscope slides. Images of stained cells were captured by fluorescence microscopy at a magnification of x200 (Leica DMI4000 B). The immunofluorescence optical density was quantified from three independent experiments using Image $\mathrm{J}$ software (version 1.3; National Institutes of Health, Bethesda, MD, USA).

Reverse transcription-quantitative polymerase chain reaction (RT-qPCR). RT-qPCR was performed to quantify the mRNA levels of ROCK1/2, ZO-1 and occludin. Total RNA was extracted from RKO cells, which were treated with $0.1 \%$ DMSO or melatonin (2 and $3 \mathrm{mM}$ ) for $48 \mathrm{~h}$ at $37^{\circ} \mathrm{C}$, using TRIzol (Invitrogen; Thermo Fisher Scientific, Inc.) according to the manufacturer's protocol. A total of $4 \mu \mathrm{g}$ of total RNA was reverse transcribed using the PrimeScript ${ }^{\mathrm{TM}} \mathrm{RT}$ reagent kit with gDNA Eraser (Takara Biotechnology Co., Ltd., Dalian, China) according to the manufacturer's protocol. The thermocycling conditions were as follows: at $42^{\circ} \mathrm{C}$ for $2 \mathrm{~min}, 37^{\circ} \mathrm{C}$ for $15 \mathrm{~min}$ and $85^{\circ} \mathrm{C}$ for $5 \mathrm{sec}$. The complementary DNAs were amplified using the SYBR-Green qPCR Master mix (Takara Biotechnology Co., Ltd.) and a Real-Time PCR system. All the primers were synthesized by the Sangon Biotech Co., Ltd. (Shanghai, China) and the sequences are listed in Table I. The thermocycling conditions were as follows: Initial denaturation at $95^{\circ} \mathrm{C}$ for $30 \mathrm{sec}$, followed by 40 cycles of $95^{\circ} \mathrm{C}$ for $5 \mathrm{sec}$ and $60^{\circ} \mathrm{C}$ for $30 \mathrm{sec}$. Relative quantification was performed using the $2^{-\Delta \Delta \mathrm{Cq}}$ method (27) and the results were normalized to those of GAPDH.

Western blot analysis. Following treatment, the RKO cells, which had be treated with DMSO, melatonin, SB203580 and PMA $(1 \mu \mathrm{M})$ for $48 \mathrm{~h}$ at $37^{\circ} \mathrm{C}$, were collected and washed three times with PBS. Total protein extracts were prepared with
Table I. Sequences of the primers used in reverse transcription-quantitative polymerase chain reaction.

\begin{tabular}{ll}
\hline Primer name & \multicolumn{1}{c}{ Primer sequences (5'-3') } \\
\hline GAPDH-F & AGGTCGGAGTCAACGGATTTG \\
GAPDH-R & CCTGGAAGATGGTGATGGGAT \\
ROCK1-F & CTCTACCACTTTCCTGCCAA \\
ROCK1-R & GTGGCACTTAACATGGCATC \\
ROCK2-F & ACCAATGCTTTACTGCGAAC \\
ROCK2-R & TCTCCAGCAGGCAGTTTTTA \\
ZO-1-F & CTCTCAACAGGTGTATAGAAAGGATCC \\
ZO-1-R & CTACGTATGGGAGTTGGGGTTC \\
Occludin-F & AGAACTCTCCCGTTTGGATAAAGA \\
Occludin-R & TTTGTAATCTGCAGATCCCTTCAC \\
\hline
\end{tabular}

F, forward; R, reverse; ROCK, Rho-associated protein kinase; ZO-1, zonula occludens- 1 .

radioimmunoprecipitation buffer [hepes $25 \mathrm{mM}, 1.5 \%$ Triton $\mathrm{X}-100,1 \%$ sodium deoxycholate, $0.1 \%$ SDS, $\mathrm{NaCl} 0.5 \mathrm{M}$, EDTA $5 \mathrm{mM}$, NaF $50 \mathrm{mM}$, sodium vanadate $0.1 \mathrm{mM}$, phenylmethylsulfonyl fluoride $1 \mathrm{mM}$, and leupeptin $0.1 \mathrm{~g} / \mathrm{l},(\mathrm{pH} 7.8)]$ and measured using a bicinchoninic protein assay kit (Beyotime Institute of Biotechnology, Haimen, China). To further verify ZO-1 and occludin expression, membrane proteins were also extracted using the Membrane, Nuclear and Cytoplasmic Protein Extraction kit (Sangon Biotech Co., Ltd.) according to the manufacturer's instruction. Cell lysates were solubilized in SDS sample buffer, separated by $8-15 \%$ SDS-PAGE with $60 \mu \mathrm{g}$ protein loaded/lane, and transferred to a PVDF membrane, which was blocked with blocking buffer ( $5 \%$ non-fat dry milk) for $2 \mathrm{~h}$ at room temperature. Membranes were then incubated with the primary antibodies against MLC, p-MLC, p-p38, p38, ROCK1, ROCK2, MYPT1, p-MYPT1, ZO-1, occludin, $\mathrm{Na}^{+} / \mathrm{K}^{+}$-ATPase and $\beta$-actin (dilution of all primary antibodies $1: 1,000)$ overnight at $4^{\circ} \mathrm{C}$, followed by incubation with the corresponding horseradish peroxidase-conjugated secondary antibody (1:3,000; EMD Millipore) for $2 \mathrm{~h}$ at room temperature. The reactive bands were visualized using ECL (Pierce; Thermo Fisher Scientific, Inc.) and a Kodak XAR film. The images were scanned using a ScanPrisa 1240UT scanner (Acer America Corporation, San Jose, CA, USA) and data were quantified from three independent experiments using Quantity One software (version 4.6.2; Bio-Rad Laboratories, Inc.). The results obtained from total proteins were normalized to those of $\beta$-actin and those generated from membrane proteins were normalized to those of $\mathrm{Na}^{+} / \mathrm{K}^{+}$-ATPase.

Statistical analysis. At least three independent experiments were performed for each experiment and the data are expressed as mean \pm standard deviation. The difference between treatment and control groups were analyzed by one-way analysis of variance followed by Dunnett's or least significant difference post hoc tests using SPSS 16.0 software (SPSS, Inc., Chicago, IL, USA). Quantitative analysis of immunofluorescence was performed using two independent samples t-tests. $\mathrm{P}<0.05$ was considered to indicate a statistically significant difference. 

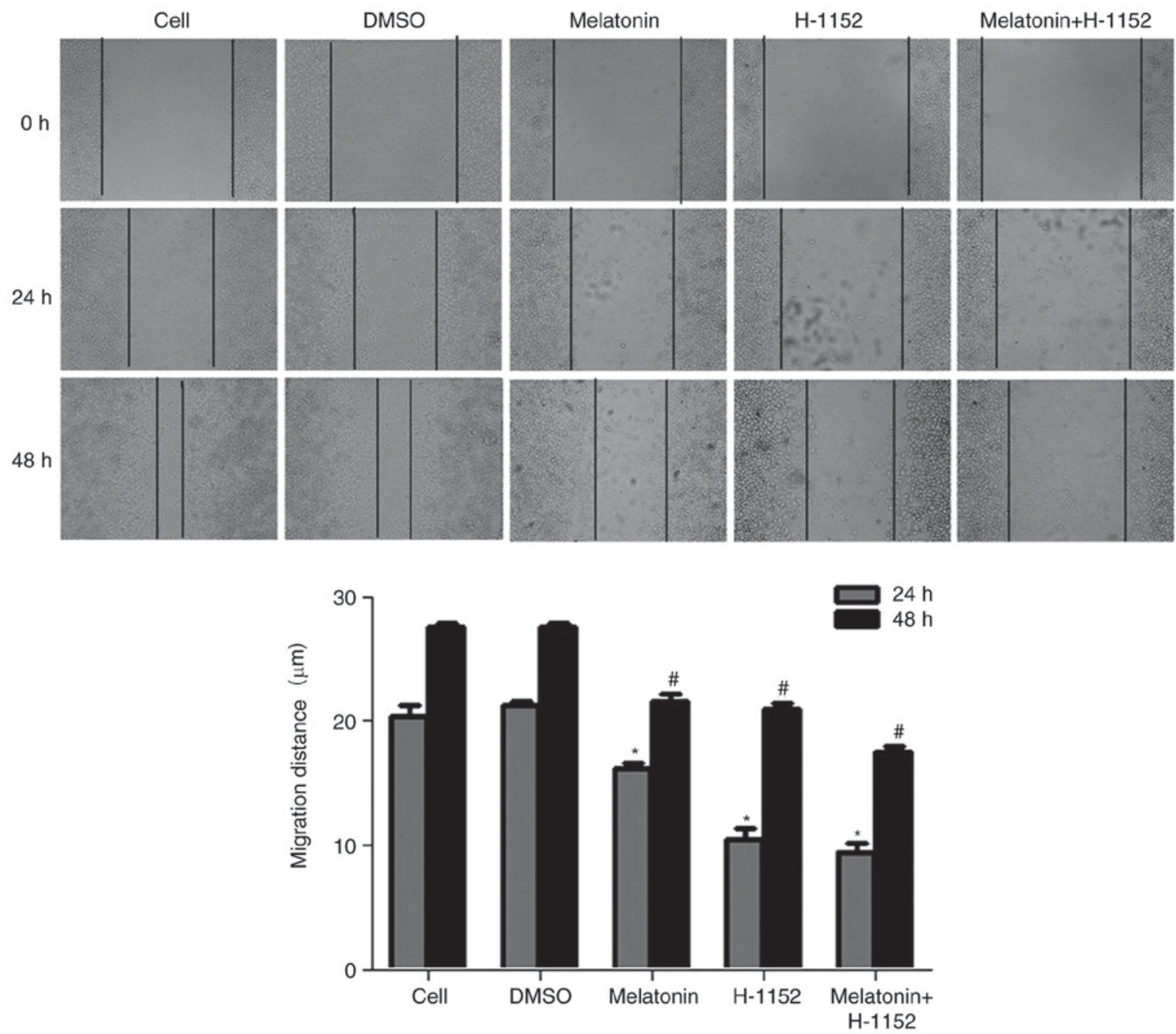

Figure 1. Effect of melatonin and H-1152 on RKO cell migration. Cell migratory ability was decreased following RKO cell treatment with melatonin (2.5 mM) and the ROCK inhibitor, H-1152 $(10 \mu \mathrm{M})$, alone or in combination, for 0,24 and $48 \mathrm{~h}$ (magnification, $\mathrm{x} 100$ ). The width of the wound was measured and the following calculation was used: Half of migration distance $=$ (end distance - start distance) $/ 2$. ${ }^{*} \mathrm{P}<0.05$ vs. the DMSO at $24 \mathrm{~h}$; $\mathrm{F}<0.05$ vs. DMSO $48 \mathrm{~h}$. H-1152, 5-[[(2s)-hexahydro-2-methyl-1H-1,4-diazepin-1-yl] sulfonyl]-4-methyl-isoquinoline, dihydrochloride.

\section{Results}

Effect of melatonin on RKO cell migration. A previous study indicated that melatonin inhibited the migration of RKO cells in a concentration-dependent manner and may also inhibit the migration of RKO cells following treatment with SB203580 and PMA in vitro via the p38 MAPK signaling pathway (8). To further explore the association between cell migration and ROCK, a wound-healing assay was performed on RKO cells treated with H-1152, a ROCK inhibitor. Following treatment with melatonin alone or in combination with H-1152 for 24 and $48 \mathrm{~h}$, the contrast half of migration distance was significantly inhibited by all melatonin and H-1152 treatments when compared with the control groups (Fig. 1 and Table II).

Melatonin decreases the expression of ROCK2 and the phosphorylation of MYPT1, and increases the expression of occludin in RKO cells. Following cell treatment with different concentrations of melatonin $(1,2$ and $3 \mathrm{mM})$ for $48 \mathrm{~h}$, the results revealed that the protein expression levels of ROCK2 and p-MYPT1 were significantly decreased in a concentration-dependent manner when compared to the DMSO control
Table II. Effects of melatonin on migration in RKO cells.

\begin{tabular}{lccc}
\hline & & \multicolumn{2}{c}{ Half migration distance $(\mathrm{mm})$} \\
\cline { 3 - 4 } $\begin{array}{l}\text { Treatment } \\
\text { group }\end{array}$ & Dose & $24 \mathrm{~h}$ & $48 \mathrm{~h}$ \\
\hline Cells in media & - & $20.379 \pm 0.855$ & $27.551 \pm 0.273$ \\
DMSO & - & $21.243 \pm 0.332$ & $27.500 \pm 0.390$ \\
Melatonin & $2.5 \mathrm{mM}$ & $16.104 \pm 0.546^{\mathrm{a}}$ & $21.522 \pm 0.568^{\mathrm{a}}$ \\
$\mathrm{H}-1152$ & $10 \mu \mathrm{M}$ & $10.482 \pm 0.844^{\mathrm{a}}$ & $20.955 \pm 0.410^{\mathrm{a}}$ \\
Melatonin + & $2.5 \mathrm{mM}+$ & $9.474 \pm 0.623^{\mathrm{a}}$ & $17.534 \pm 0.453^{\mathrm{a}}$ \\
$\mathrm{H}-1152$ & $10 \mu \mathrm{M}$ & & \\
\hline
\end{tabular}

Data are presented as the mean \pm standard deviation $(\mathrm{n}=5) .{ }^{\mathrm{a}} \mathrm{P}<0.05$ vs. DMSO. DMSO, dimethyl sulfoxide; H-1152, 5-[[(2s)-hexahydro-2methyl-1H-1, 4-diazepin-1-yl] sulfonyl]-4-methyl-isoquinoline, dihydrochloride.

treatment (Fig. 2A and B). In addition, the present study also assessed the effect of melatonin on the mRNA expression 
A
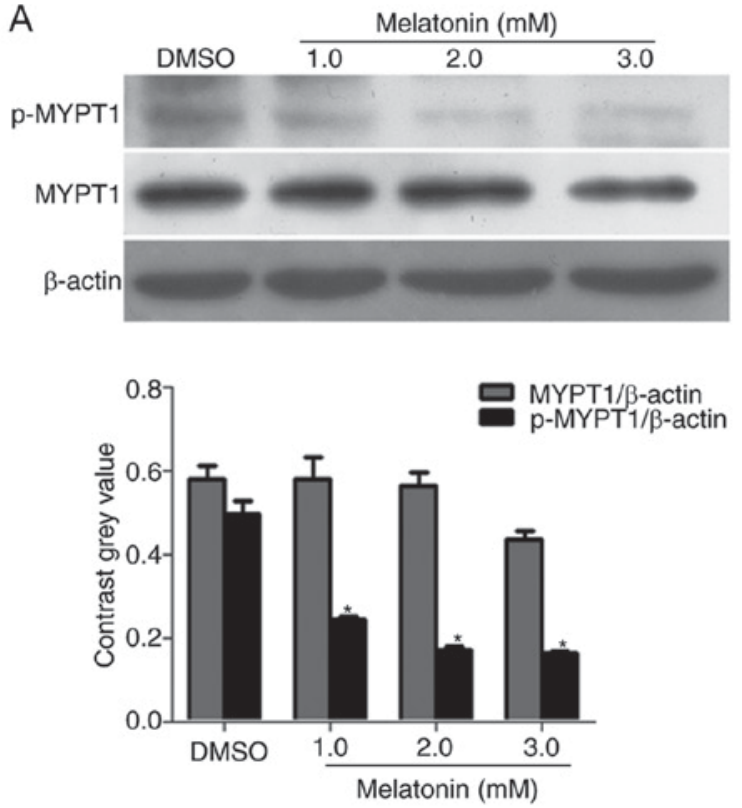

C

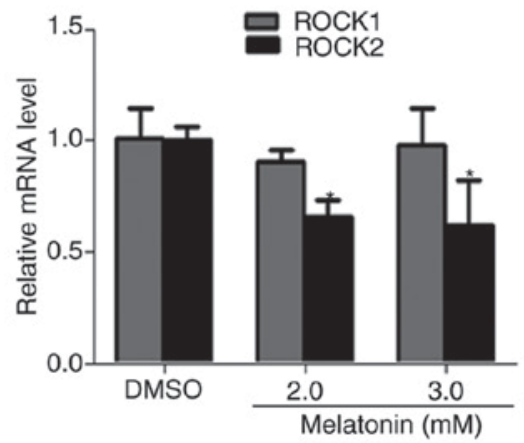

E
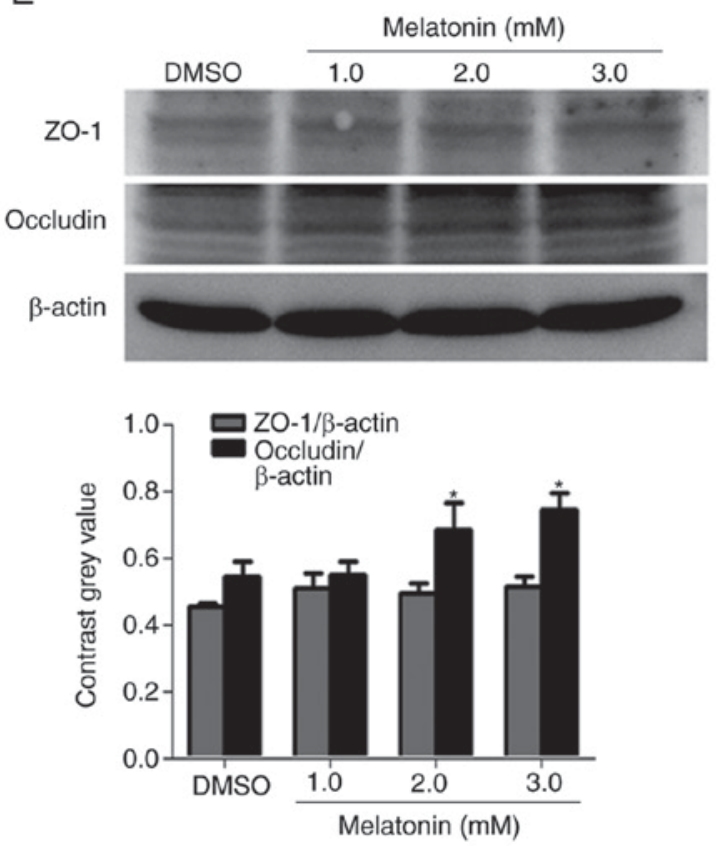

B
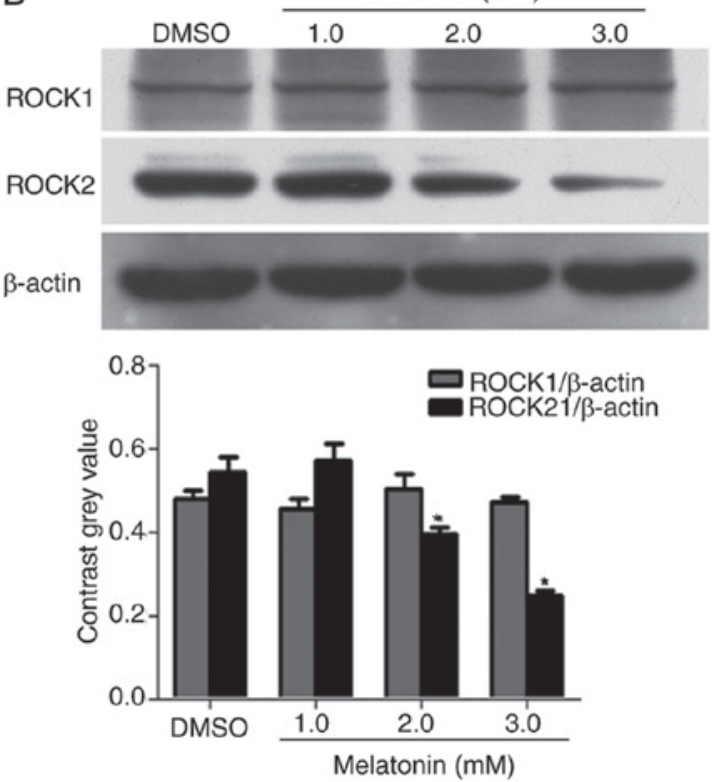

D

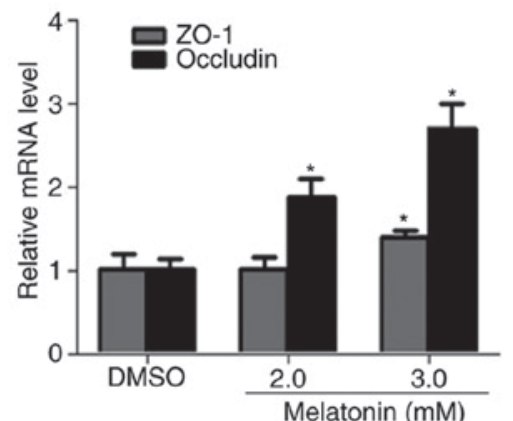

F
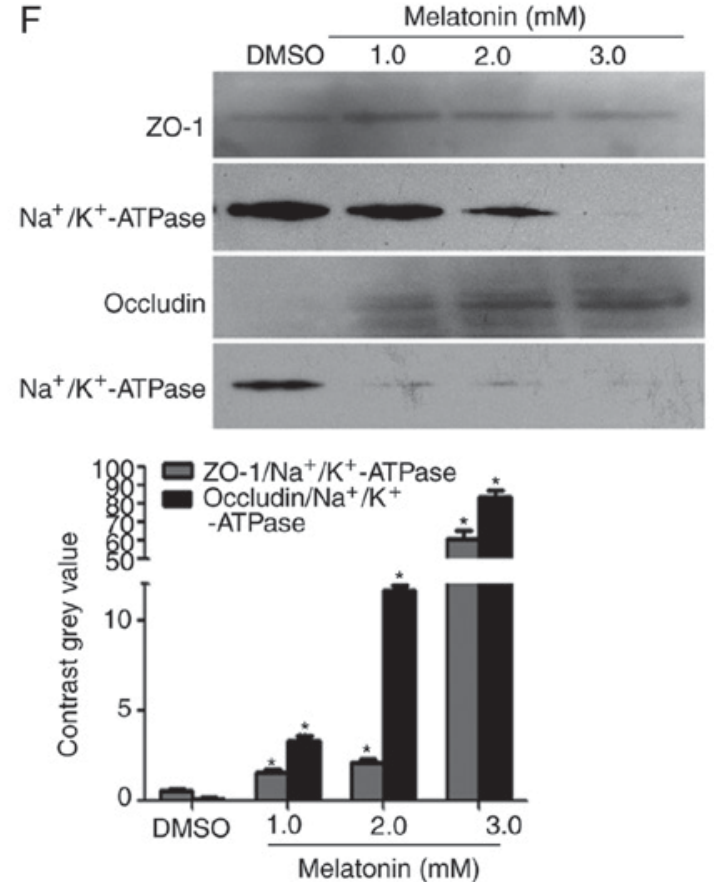

Figure 2. Effect of melatonin on ROCK expression and the phosphorylation of MYPT1. Western blotting was performed to determine the protein expression of (A) MYPT1 and p-MYPT1, and (B) ROCK1/2 in RKO cells. Treatment for $48 \mathrm{~h}$ with different concentrations $(1,2$ and $3 \mathrm{mM})$ of melatonin markedly decreased the protein expression of ROCK2 and p-MYPT1 in RKO cells. Reverse transcription-quantitative polymerase chain reaction was performed to evaluate the relative mRNA levels of (C) ROCK1/2 and (D) ZO-1 and occludin. The mRNA expression of ROCK2 was decreased with melatonin, which reflected the results of western blotting. In addition, the mRNA expression of ZO-1 and occludin were increased with melatonin treatment. (E) In the total proteins, melatonin only significantly increased the protein expression of occludin; treatment had no effect on ZO-1 expression. (F) However, in the membrane proteins the expression of ZO-1 and occludin was significantly increased when compared with the control group. " $\mathrm{P}<0.05$ vs. the DMSO group. ROCK, Rho-associated protein kinase; p-, phospho-; MYPT1, myosin phosphatase targeting subunit 1; ZO-1, zona occludens-1. 
Table III. Effects of melatonin on the mRNA expression levels in RKO cells.

Relative mRNA expression

\begin{tabular}{lccccc}
\cline { 3 - 5 } Treatment group & Dose $(\mathrm{mM})$ & ROCK1 & ROCK2 & ZO-1 & Occludin \\
\hline DMSO & - & $1.007 \pm 0.142$ & $1.001 \pm 0.060$ & $1.010 \pm 0.177$ & $1.007 \pm 0.136$ \\
Melatonin & 2.0 & $0.908 \pm 0.052$ & $0.659 \pm 0.076^{\mathrm{a}}$ & $1.012 \pm 0.147$ & $1.877 \pm 0.214^{\mathrm{a}}$ \\
& 3.0 & $0.982 \pm 0.162$ & $0.623 \pm 0.202^{\mathrm{a}}$ & $1.391 \pm 0.089^{\mathrm{a}}$ & $2.703 \pm 0.280^{\mathrm{a}}$
\end{tabular}

Data are presented as the mean \pm standard deviation $(n=3) . ~{ }^{\mathrm{P}}<0.05$ vs. DMSO. DMSO, dimethyl sulfoxide; ROCK, Rho-associated protein kinase; ZO-1, zonula occludens-1.

of ROCK and several TJ proteins in RKO cells by qPCR and western blotting. ROCK1 expression was not altered at the mRNA and protein levels, however, ROCK2 mRNA and protein was significantly downregulated by melatonin when compared with DMSO treatment (Fig. 2B and C). Melatonin also increased the mRNA level of ZO-1 and occludin in a concentration-dependent manner (Fig. 2D and Table III). In addition, the protein expression levels of occludin were significantly increased with high concentration of melatonin $(3 \mathrm{mM})$, however, ZO-1 was only marginally increased with melatonin treatment (Fig. 2E). To further confirm this, membrane proteins were isolated using a Membrane, Nuclear and Cytoplasmic Protein Extraction kit. The results demonstrated that the membrane protein expression of $\mathrm{ZO}-1$ and occludin were significantly upregulated following melatonin treatment when the results were normalized to those of $\mathrm{Na}^{+} / \mathrm{K}^{+}$-ATPase instead of the $\beta$-actin level (Fig. 2F).

As shown in Fig. 3, following treatment with melatonin and $\mathrm{H}-1152$, alone or in combination, for $48 \mathrm{~h}$, the expression of p-MYPT1, ROCK2 and p-MLC were significantly decreased, however, the expression of MYPT1, ROCK1 and MLC were not altered (Fig. 3A-C). Our previous study (8) suggested that the phosphorylation of $\mathrm{p} 38$ was inhibited by melatonin in a concentration-dependent manner. The present study further determined p-p38 expression and revealed that when compared with the control group, p38 phosphorylation was downregulated in cells treated with $\mathrm{H}-1152$ for $4 \mathrm{~h}$ (Fig. 3D).

Melatonin increases the expression of ZO-1 and occludin in $R K O$ cell TJS. In order to detect the expression and localization of ZO-1 and occludin proteins, an immunofluorescence assay was performed. Following RKO cell treatment with melatonin, the fluorescence intensity of ZO-1 and occludin was increased, particularly in regions with TJs, when compared with the control group (Fig. 4A and B). Quantitative analysis of immunofluorescence revealed that the expression of ZO-1 and occludin was increased in the melatonin-treated group, which was consistent with the western blotting results (Fig. 4C and D).

Melatonin downregulates the expression of ROCK via the p38/MAPK signaling pathway in RKO cells. Following the aforementioned experiments, it was speculated whether the expression and activity of ROCK may be relevant in p38 MAPK signaling. Therefore, the effect of PMA, a PKC activator, and SB203580, a p38 inhibitor, on the expression of p-MYPT1 was determined (Fig. 5). Western blot analysis demonstrated that melatonin inhibited the expression of the p-MYPT1. In addition, treatment with melatonin and SB combined further reduced the expression levels of p-MYPT1. However, although the expression of p-MYPT1 was significantly decreased when cells were treated in combination with PMA compared with PMA treatment alone, this decrease was not as marked as that observed with melatonin and SB treatments.

\section{Discussion}

The hallmarks of cancer include sustaining proliferative signaling, evading growth suppressors, resisting cell death, enabling replicative immortality, inducing angiogenesis, and activating cell invasion and metastasis (28). Recently, several studies have indicated that melatonin can modulate microtubule and microfilament structure formation, and suppress the invasive and metastatic potential of invasive and metastatic potential of breast (6), colon (8), liver (10) and lung (13) cancer cells via different signaling pathways. However, the underlying mechanism of melatonin on RKO cells migration inhibition is poorly understood. In the present study, melatonin suppressed the migration of human colon cancer RKO cells, potentially via the P-38/MAPK signaling pathway, and also decreased the expression of ROCK and TJ associated proteins.

The two ROCK isoforms, ROCK1 and ROCK2, which share 65\% homology in their amino acid sequences and $92 \%$ homology in their kinase domains, are highly homologous. Although the two isoforms are ubiquitously expressed, the expression of ROCK 1 and ROCK 2 are more prominent in different tissues including the liver and lung, and the brain and heart, respectively $(29,30)$. In the present study, the data demonstrated that the protein expression of ROCK2 in RKO cells treated with various concentrations of melatonin was downregulated. In addition, the mRNA levels of ROCK2 were suppressed with increasing concentrations of melatonin, however, the expression of ROCK1 was not altered. These results indicate that melatonin may reduce ROCK 2 expression at the transcriptional and protein level. In the author's previous study (8), the phosphorylation of MYPT1 and MLC was inhibited by melatonin in a concentration-dependent manner. Therefore, ROCK2 may serve a role in the dephosphorylation of MLC, as opposed to ROCK1, in RKO cells. There is an increasing body of evidence that has indicated that ROCK may be active and augment tumor aggressive properties such as metastasis and invasion in gastric cancer cells $(31,32)$. Thus, a reduction in ROCK activity may 

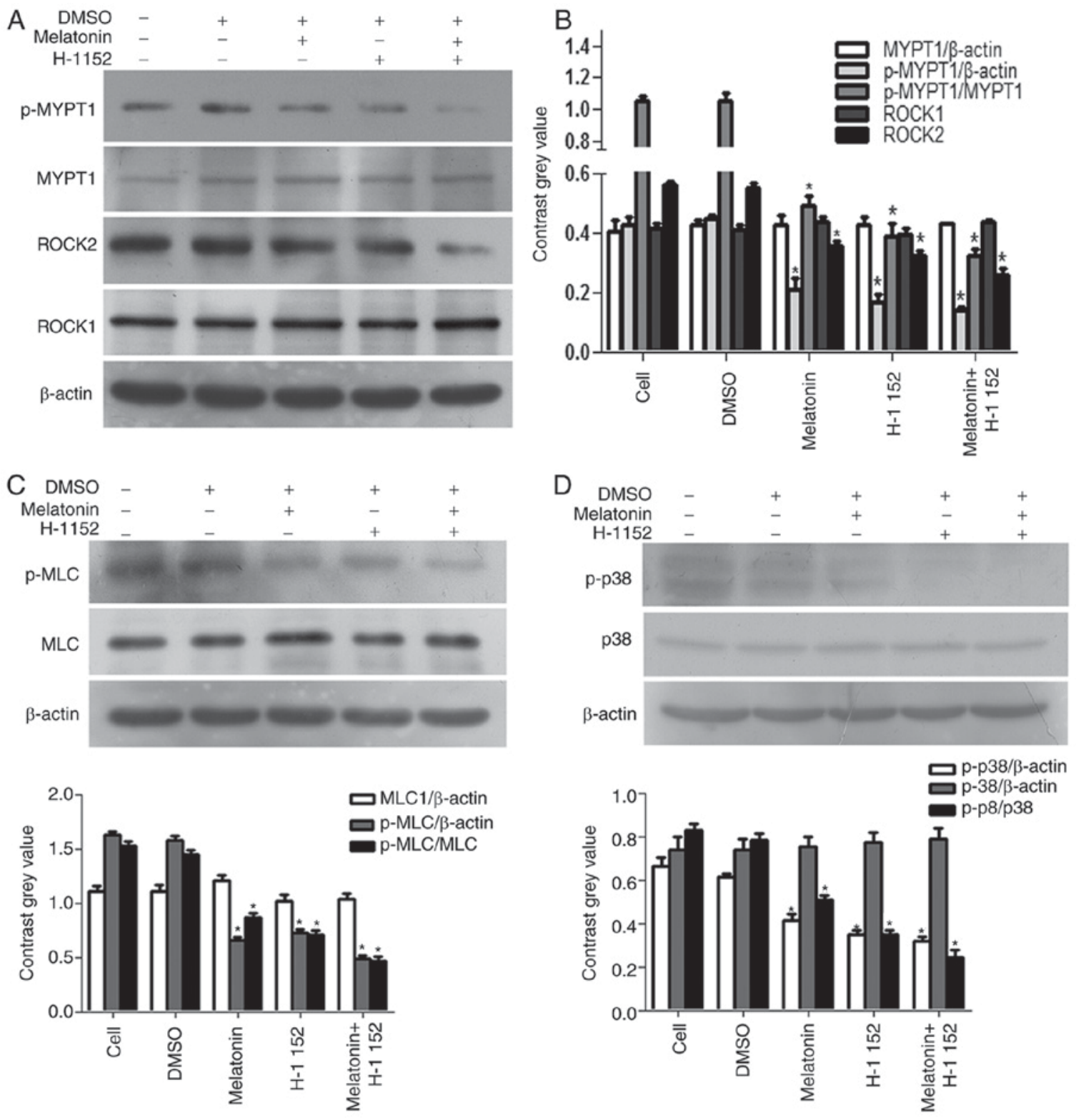

Figure 3. Effect of melatonin and H-1152 on ROCK expression, and the phosphorylation of MYPT1 and p38. H-1152 (10 $\mu \mathrm{M})$ was used to treat RKO cells, alone and in combination with melatonin, and the expression of (A) p-MYPT1 and ROCK2 were significantly decreased. (B) Quantification of the protein expression levels of p-MYPT1 and ROCK2. (C) p-MLC was significantly decreased. (D) In addition, the phosphorylation of p38 was also decreased in RKO cells following H-1152 treatment, alone or in combination with melatonin $(2.5 \mathrm{mM})$ for $4 \mathrm{~h}$, thereby exhibiting the same trend as ROCK 2 expression. ${ }^{*} \mathrm{P}<0.05$ vs. the DMSO group. H-1152, 5-[[(2s)-hexahydro-2-methyl-1H-1,4-diazepin-1-yl] sulfonyl]-4-methyl-isoquinoline, dihydrochloride; ROCK, Rho-associated protein kinase; MYPT1, myosin phosphatase targeting subunit 1; p-, phospho-; MLC, myosin light chains.

inhibit the migration and invasion of cancer cells. Despite their extensive homology and substrate promiscuity, the two ROCK isoforms have distinct functions. Notably, ROCK2 depletion has been reported to enhance microfilament bundle assembly into stress fibers and the formation of focal adhesion, while ROCK1-depleted cells exhibit the opposite phenotype (33).

Our previous findings suggested that the migration of RKO cells was inhibited in a concentration-dependent manner following treatment with melatonin (8). To confirm the association between ROCK and RKO cell migration, the present study treated cells with H-1152 to inhibit ROCK function, which demonstrated that RKO cell migration was markedly inhibited when compared with the control group. H-1152, a specific inhibitor of ROCK, also decreased ROCK protein expression; ROCK2 protein expression and the phosphorylation of MYPT1 were inhibited following treatment with melatonin and H-1152, alone or in combination. In addition, the phosphorylation level of MLC was also decreased. These results indicate that melatonin may exert inhibitory effects on RKO cell migration by attenuating the expression of ROCK2 and the phosphorylation of MLC. Previous studies have reported that MLC phosphorylation is essential to initiate actin-myosin interactions. Phosphorylation of MLC activates myosin ATPase activity, which couples with actin-myosin filaments to the plasma membrane, thereby increasing the generation of actin-myosin contractile force and cell contractility $(8,11)$. Pretreatment with the specific ROCK inhibitor, Y27632, has also been revealed to abrogate MLC phosphorylation and suppress membrane contraction (34). 

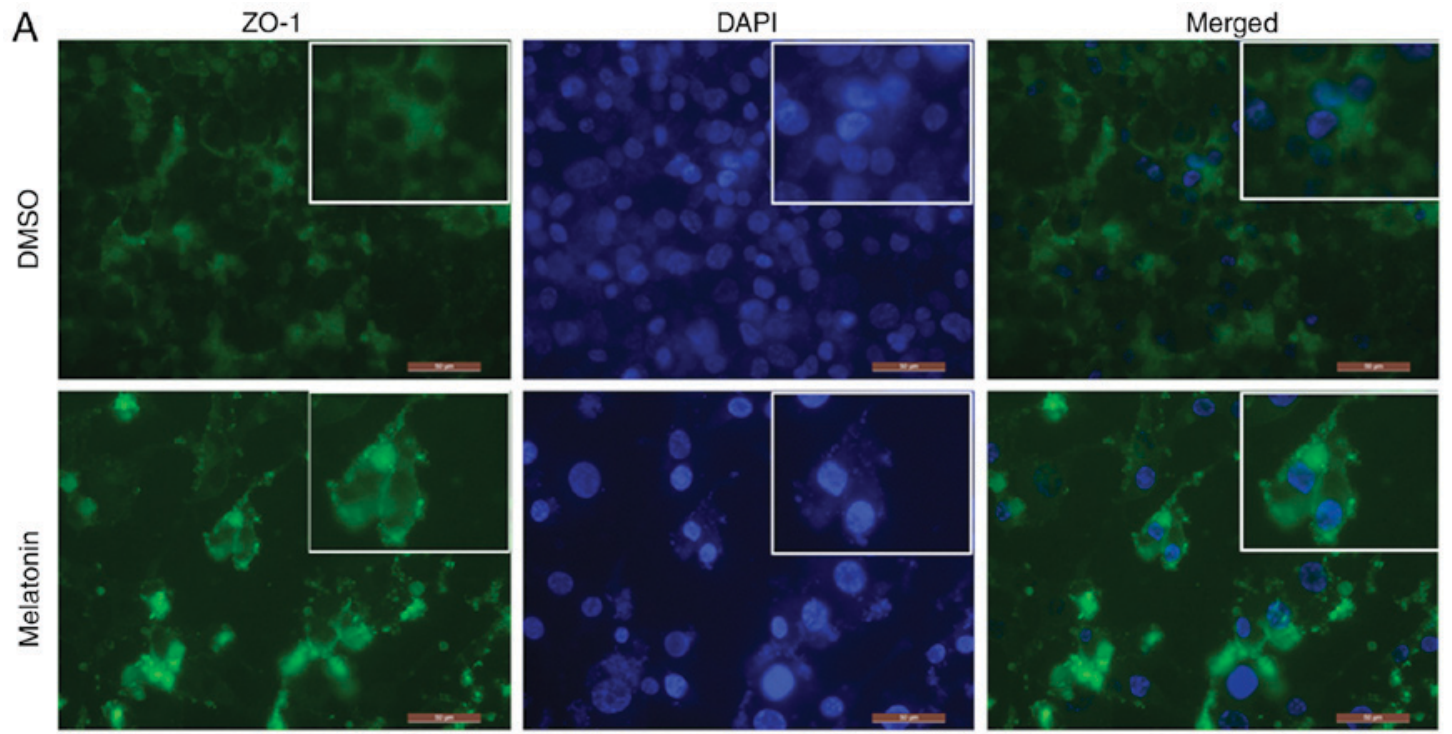

B

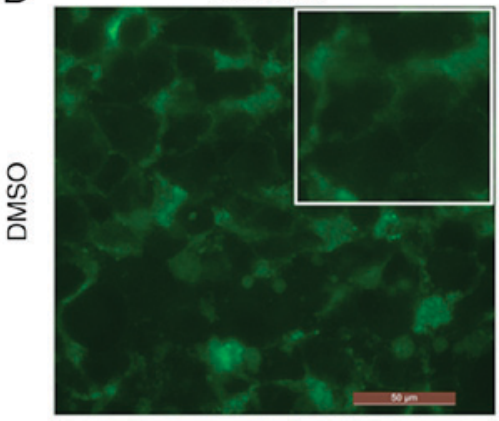

DAPI
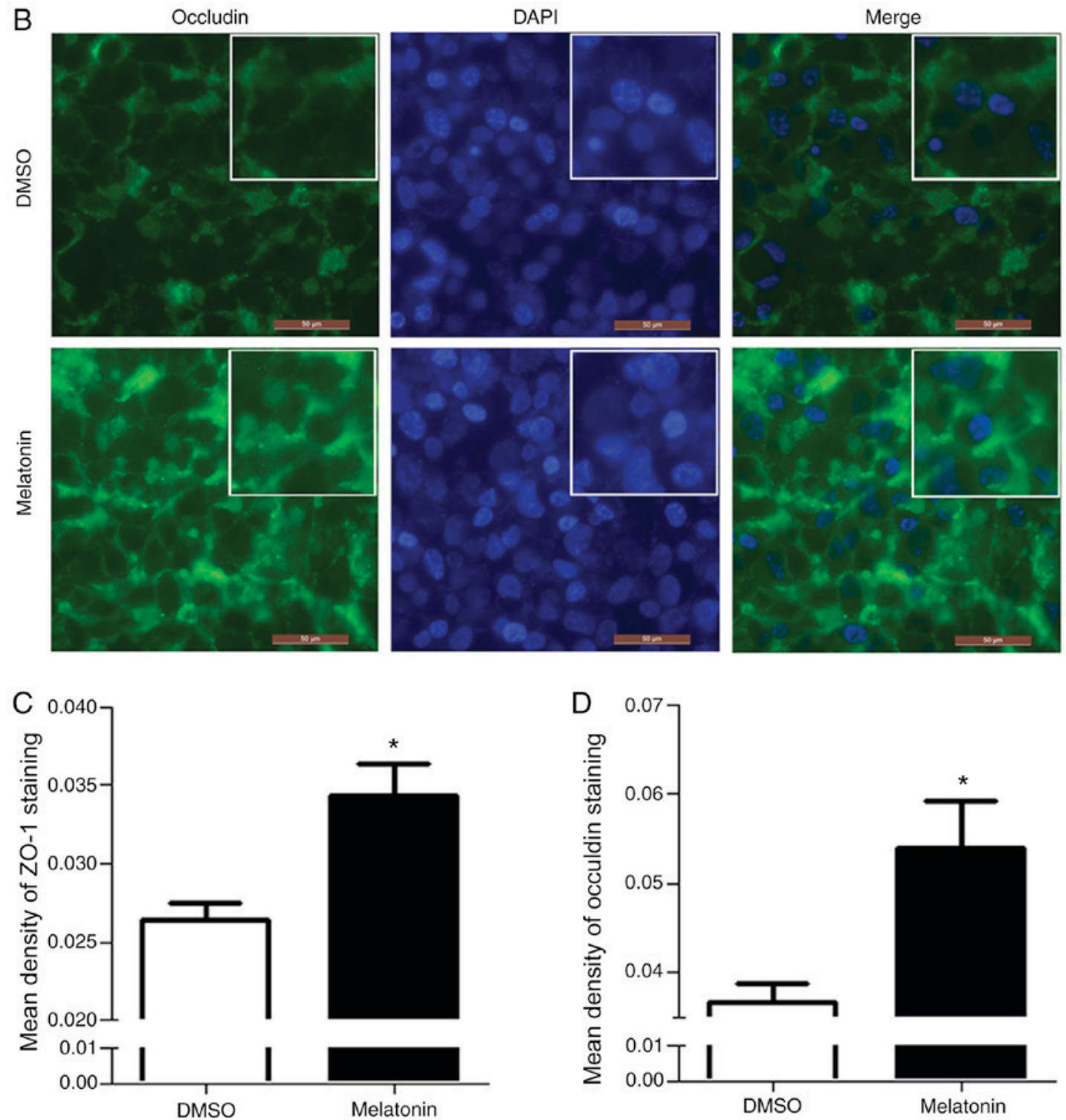

Figure 4. Melatonin increases the expression and localization of ZO-1 and occludin in RKO cell tight junctions. The position of (A) ZO-1 and (B) occludin were observed by an immunofluorescence assay. Following RKO cell treatment with melatonin ( $2.5 \mathrm{mM})$ for 10 days, then nuclei were counterstained with DAPI (blue), and ZO-1 and occludin were stained with FITC (green). Staining was photographed using a laser scanning confocal microscope (magnification, x200; scale bar, $50 \mu \mathrm{m}$ ). Quantitative analysis of immunofluorescence revealed that melatonin induced the expression of (C) ZO-1 and (D) occludin; this reflects the western blotting results. Two independent sample t-tests were performed for analyses. " $\mathrm{P}<0.05$ vs. the DMSO group. DAPI, 4',6-diamidino-2-phenylindole; FITC, fluorescein isothiocyanate; ZO-1, zona occludens-1. 

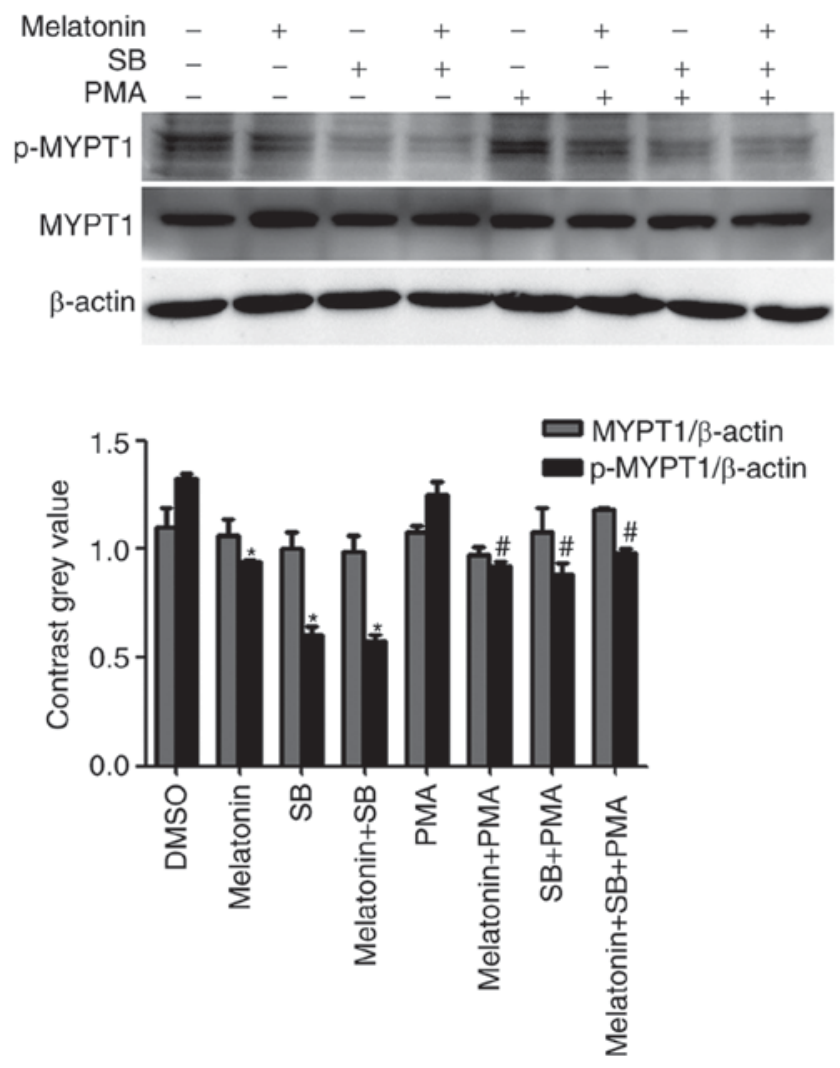

Figure 5. Effect of melatonin, SB203580 and PMA on the expression of p-MYPT1. Cells were treated with DMSO, or with melatonin $(2.5 \mathrm{mM})$, SB203580 $(10 \mu \mathrm{M})$ and PMA $(1 \mu \mathrm{M})$, alone or in combination, for $48 \mathrm{~h}$. The expression of p-MYPT1 decreased when cells were exposed to SB203580. Combined treatment of melatonin and/or SB203580 with PMA inhibited the phosphorylation of MYPT1 however, the decrease in p-MYPT1 expression observed was not as marked. Thus, PMA partially prevented the melatonin or SB203580-induced decrease in p-MYPT1 expression. ${ }^{*} \mathrm{P}<0.05$, vs. the DMSO group; ${ }^{\text {}} \mathrm{P}<0.05$, vs. the PMA only group. PMA, Phorbol 12 -myristate 13-acetate; p-, phospho-; MYPT1, myosin phosphatase targeting subunit 1; SB, SB203580.

In the present study, the membrane proteins ZO-1 and occludin were observed to be increased in RKO cells treated with melatonin. Immunofluorescence assay analyses also revealed that melatonin induced the localized expression of ZO-1 and occludin at cell TJs. In addition, some studies have also suggested that melatonin may inhibit tumor invasion by increasing the expression of TJ-associated proteins $\mathrm{ZO}-1$ and occludin $(13,14)$. These results suggest that translocation of ZO-1 and occludin may contribute to the anti-migration effect of melatonin.

Our previous study demonstrated that the phosphorylation of p38 was decreased by melatonin treatment in a concentration-dependent manner (8). In addition, p38 MAPK has been reported to be a key signaling molecule in the regulation of cancer invasion and metastasis $(35,36)$. In the present study, western blotting suggested that the phosphorylation of p38 was suppressed by H-1152, thus exhibiting a similar trend to that of ROCK2 expression. Therefore, ROCK 2 may contribute to the migration of RKO cells by inhibiting p38 MAPK. The authors' previous study verified that SB203580, an inhibitor of the p38 MAPK signaling pathway, further enhanced the melatonin-induced inhibition of RKO migration, while PMA, a PKC activator, weakened this inhibitory effect (8), the inhibitory effect of SB203580 was demonstrated to be similar to H-1152-mediated migration. The present study confirmed that the expression of p-MYPT1 was more markedly reduced following the combined treatment with melatonin and SB203580 when compared with melatonin treatment alone, while the combined treatment with PMA attenuated this inhibitory effect. In addition, SB203580 markedly decreased the expression of p-MYPT1 same as p-p38 in the PMA-stimulated group in the authors' previous study. Due to its inhibitory effect on p38 phosphorylation, previous studies have suggested that melatonin is a promising anti-invasion factor that may also be effective in cancer therapies targeting breast cancer $(37,38)$; this is consistent with the results of the present study. Collectively, these results indicate that the anti-migration effect of melatonin may be associated with the downregulation of the p38 MAPK signaling pathway and ROCK expression.

In conclusion, melatonin inhibited the migration of RKO cells by decreasing ROCK expression, and increasing the expression of ZO-1 and occludin on the cell membrane, which may be associated, at least in part, with the p38 MAPK signaling pathway. These results provide evidence for the potential clinical application of melatonin in the treatment of metastatic colorectal cancers. However, tumor cell migration and the formation of tumor metastases are extremely complex processes, thus further studies are required to elucidate the comprehensive molecular mechanisms underlying melatonin-induced inhibition of tumor cell migration.

\section{Acknowledgements}

The present study was funded by the Science Foundation for the Excellent Youth Scholars of Universities of Anhui Province (grant no. 2013SQRL101ZD), the Anhui Natural Science Foundation (grant no. 1508085QH167) and the National Natural Science Foundation of China (grant no. 81272399).

\section{References}

1. André T, Boni C, Mounedji-Boudiaf L, Navarro M, Tabernero J, Hickish T, Topham C, Zaninelli M, Clingan P, Bridgewater J, et al: Oxaliplatin, fluorouracil, and leucovorin as adjuvant treatment for colon cancer. N Engl J Med 350: 2343-2351, 2004.

2. Vellinga TT, Borovski T, de Boer VC, Navarro M, Tabernero J, Hickish T, Topham C, Zaninelli M, Clingan P, Bridgewater J, et al: SIRT1/PGCl $\alpha$ dependent increase in oxidative phosphorylation supports chemotherapy resistance of colon cancer. Clin Cancer Res 21: 2870-2879, 2015.

3. Akbulut KG, Aktas SH and Akbulut $\mathrm{H}$ : The role of melatonin, sirtuin 2 and FoXO1 transcription factor in the aging process of colon in male rats. Biogerontol 16: 99-108, 2015.

4. Xu C, Wu A, Zhu H, Fang H, Xu L, Ye J and Shen J: Melatonin is involved in the apoptosis and necrosis of pancreatic cancer cell line SW-1990 via modulating of Bcl-2/Bax balance. Biomed Pharmacother 67: 133-139, 2013.

5. Wang J, Guo W, Chen W, Yu W, Tian Y, Fu L, Shi D, Tong B, Xiao X, Huang W and Deng W: Melatonin potentiates the antiproliferative and pro-apoptotic effects of ursolic acid in colon cancer cells by modulating multiple signaling pathways. J Pineal Res 54: 406-416, 2013.

6. Mao L, Yuan L, Slakey LM, Jones FE, Burow ME and Hill SM: Inhibition of breast cancer cell invasion by melatonin is mediated through regulation of the p38 mitogen-activated protein kinase signaling pathway. Breast Cancer Res 12: R107, 2010.

7. Yi C, Zhang Y, Yu Z, Xiao Y, Wang J, Qiu H, Yu W, Tang R, Yuan Y, Guo W and Deng W: Melatonin enhances the antitumor effect of fisetin by inhibiting COX-2/iNOS and NF-kB/p300 signaling pathways. PLoS One 9: 99943, 2014. 
8. Zou DB, Wei X, Hu RL, Yang XP, Zuo L, Zhang SM, Zhu HQ, Zhou Q, Gui SY and Wang Y: Melatonin inhibits the migration of colon cancer RKO cells by down-regulating myosin light chain kinase expression through cross-talk with p38 MAPK. Asian Pac J Cancer Prev 16: 5835-5842, 2015.

9. Benítez-King G, Soto-Vega E and Ramírez-Rodriguez G: Melatonin modulates microfilament phenotypes in epithelial cells: Implications for adhesion and inhibition of cancer cell migration. Histol Histopathol 24: 789-799, 2009.

10. Ordoñez R, Carbajo-Pescador S, Prieto-Dominguez N, García-Palomo A, González-Gallego J and Mauriz JL: Inhibition of matrix metalloproteinase-9 and nuclear factor kappa B contribute to melatonin prevention of motility and invasiveness in HepG2 liver cancer cells. J Pineal Res 56: 20-30, 2014.

11. Olson MF and Sahai E: The actin cytoskeleton in cancer cell motility. Clin Exp Metastasis 26: 273-287, 2009.

12. Runkle EA and Mu D: Tight junction proteins: From barrier to tumorigenesis. Cancer Lett 337: 41-48, 2013.

13. Zhou Q, Gui S, Zhou Q and Wang Y: Melatonin inhibits the migration of human lung adenocarcinoma A549 cell lines involving JNK/MAPK pathway. PLoS One 9: 101132, 2014.

14. Hoover KB, Liao SY and Bryant PJ: Loss of the tight junction MAGUK ZO-1 in breast cancer: Relationship to glandular differentiation and loss of heterozygosity. Am J Pathol 153: 1767-1773, 1998.

15. Vicente-Manzanares M, Ma X, Adelstein RS and Horwitz AR Non-muscle myosin II takes centre stage in cell adhesion and migration. Nat Rev Mol Cell Biol 10: 778-790, 2009.

16. González-Marisca L, Tapia R and Chamorro D: Crosstalk of tight junction components with signaling pathways. Biochim Biophys Acta 1778: 729-756, 2008.

17. Ito M, Nakano T, Erdodi F and Hartshorne DJ: Myosin phosphatase: Structure, regulation and function. Mol Cell Biochem 259: 197-209, 2004

18. Pellegrin S and Mellor H: Actin stress fibres. J Cell Sci 120 3491-3499, 2007.

19. Tsuji T, Ishizaki $T$, Okanoto $M$, Higashida $C$, Kimura $K$, Furuyashiki T, Arakawa Y, Birge RB, Nakamoto T, Hirai H and Narumiya S: ROCK and mDial antagonize in Rho-dependent Rac activation in Swiss 3T3 fibroblasts. J Cell Biol 157: 819-830, 2002

20. Wojciak-Stothard B and Ridley AJ: Shear stress-induced endothelial cell polarization is mediated by Rho and Rac but not Cdc42 or PI 3-kinases. J Cell Biol 161: 429-439, 2003.

21. Totsukawa G, Wu Y, Sasaki Y, Hartshorne DJ, Yamakita Y Yamashiro S and Matsumura F: Distinct roles of MLCK and ROCK in the regulation of membrane protrusions and focal adhesion dynamics during cell migration of fibroblasts. J Cell Biol 164: 427-439, 2004.

22. Banerjee S and McGee DW: ROCK activity affects IL-1-induced signaling possibly through MKK4 and p38 MAPK in Caco-2 cells. In Vitro Cell Dev Biol Anim 52: 878-884, 2016.

23. Chang L and Karin M: Mammalian MAP kinase signaling cascades. Nature 410: 37-40, 2001

24. Cumaoglu A, Dayan S, Agkaya AO, Ozkul Z and Ozpozan NK: Synthesis and pro-apoptotic effects of new sulfonamide derivatives via activating p38/ERK phosphorylation in cancer cells. J Enzyme Inhib Med Chem 30: 413-419, 2015.
25. Grossi V, Peserico A, Tezil T and Simone C: p38 $\alpha$ MAPK pathway: A key factor in colorectal cancer therapy and chemoresistance. World J Gastroenterol 20: 9744-9758, 2014.

26. Hill SM, Belancio VP, Dauchy RT, Xiang S, Brimer S, Mao L, Hauch A, Lundberg PW, Summers W, Yuan L, et al: Melatonin: An inhibitor of breast cancer. Endocr Relat Cancer 22: R183-R204, 2015.

27. Livak KJ and Schmittgen TD: Analysis of relative gene expression data using real-time quantitative PCR and the 2(-Delta Delta C(T)) method. Methods 25: 402-408, 2001

28. Hanahan D and Weinberg RA: Hallmarks of cancer: The next generation. Cell 144: 646-674, 2011

29. Nakagawa O, Fujisawa K, Ishizaki T, Saito Y, Nakao K and Narumiya S: ROCK-I and ROCK-II, two isoforms of Rho-associated coiled-coil forming protein serine/threonine kinase in mice. FEBS Lett 392: 189-193, 1996.

30. Hahmann C and Schroeter T: Rho-kinase inhibitors as therapeutics: From pan inhibition to isoform selectivit. Cell Mol Life Sci 67: 171-177, 2010.

31. Matsuoka T, Yashiro M, Kato Y, Shinto O, Kashiwagi S and Hirakawa K: RhoA/ROCK signaling mediates plasticity of scirrhous gastric carcinoma motility. Clin Exp Metastasis 28: 627-636, 2011.

32. Liu N, Bi F, Pan Y, Sun L, Xue Y, Shi Y, Yao X, Zheng Y and Fan D: Reversal of the malignant phenotype of gastric cancer cells by inhibition of RhoA expression and activity. Clin Cancer Res 10: 6239-6247, 2004.

33. Zucchini C, Martinelli M, De Sanctis P, Rodia MT, Mattei G, Ugolini G, Montroni I, Ghignone F and Solmi R: Possible gender-related modulation by the ROCK 1 gene in colorectal cancer susceptibility. Pathobiology 82: 252-258, 2015.

34. Hsu HH, Kuo WW, Day CH, Shibu MA, Li SY, Chang SH, Shih HN, Chen RJ, Viswanadha VP, Kuo YH and Huang CY: Taiwanin E inhibits cell migration in human LoVo colon cancer cells by suppressing MMP-2/9 expression via p38 MAPK pathway. Environ Toxicol 32: 2021-2031, 2017.

35. Shin I, Kim S, Song H, Kim HR and Moon A: H-Ras-specific activation of Rac-MKK3/6-p38 pathway: Its critical role in invasion and migration of breast epithelial cells. J Biol Chem 280 14675-14683, 2005.

36. Mao L, Yuan L, Slakey LM, Jones FE, Burow ME and Hill SM: Inhibition of breast cancer cell invasion by melatonin is mediated through regulation of the p38 mitogen-activated protein kinase signaling pathway. Breast Cancer Res 2: R107, 2010.

37. Hill SM, Belancio VP, Dauchy RT, Xiang S, Brimer S, Mao L, Hauch A, Lundberg PW, Summers W, Yuan L, et al: Melatonin: An inhibitor of breast cancer. Endocr Relat Cancer 22: R183-R204, 2015.

38. Lai JM, Hsieh CL and Chang ZF: Caspase activation during phorbol ester-induced apoptosis requires ROCK dependent myosin-mediated contraction. J Cell Sci 116: 3491-3501, 2003. 